\title{
A universal graphitic interlayered centroid intercalation theory for intercalation chemistry
}

Kemeng Ji ( $\nabla$ kmji@tju.edu.cn )

Tianjin University https://orcid.org/0000-0002-4148-7252

Kailong Hu

Harbin Institute of Technology

Yuhao Shen

East China Normal University

Yoshikazu Ito

University of Tsukuba https://orcid.org/0000-0001-8059-8396

Cheng Liu

Tianjin University

Zhaozhao Liu

Tianjin University

Jiang Wang

Tianjin University

Yang Zhang

Tianjin University

Mengqian Wu

Tianjin University

Xinyu Liu

Tianjin University

Jie Sun

Tianjin University

Mingming Chen

Tianjin University

Sheng Zhang

Tianjin University https://orcid.org/0000-0001-7532-1923

Jun-ichi Fujita

University of Tsukuba

Chengyang Wang

Tianjin University 
Keywords: intercalation chemistry, Rüdorff-Hofmann model, batteries, energy

Posted Date: June 22nd, 2021

DOI: https://doi.org/10.21203/rs.3.rs-401034/v1

License: (c) (i) This work is licensed under a Creative Commons Attribution 4.0 International License. Read Full License 


\section{A universal graphitic interlayered centroid intercalation theory for intercalation chemistry}

Kemeng $\mathrm{Ji}^{1}{ }^{1}$, Kailong $\mathrm{Hu}^{2,4}$, Yuhao Shen ${ }^{3}$, Yoshikazu $\mathrm{Ito}^{4}$, Cheng Liu ${ }^{1}$, Zhaozhao Liu ${ }^{1}$, Jiang Wang $^{1}$, Yang Zhang ${ }^{1}$, Mengqian Wu ${ }^{1}$, Xinyu Liu ${ }^{1}$, Jie Sun ${ }^{1}$, Mingming Chen ${ }^{1}$, Sheng Zhang ${ }^{1}$, Jun-ichi Fujita ${ }^{2}$, Chengyang Wang ${ }^{1}$

\section{Affiliations:}

${ }^{1}$ Key Laboratory for Green Chemical Technology of Ministry of Education, School of Chemical Engineering and Technology, Tianjin University; Tianjin, 300350, P. R. China

${ }^{2}$ School of Materials Science and Engineering, Harbin Institute of Technology; Shenzhen, $10 \quad 518055$, P. R. China

${ }^{3}$ State Key Laboratory of Precision Spectroscopy and Key Laboratory of Polar Materials and Devices, Ministry of Education, Department of Electronics, East China Normal University; Shanghai, 200241, P. R. China

${ }^{4}$ Institute of Applied Physics, Graduate School of Pure and Applied Sciences, University of Tsukuba; Tsukuba, 305-8573, Japan

*Corresponding author. Email: kmji@tju.edu.cn 


\begin{abstract}
Neither of the two widely used staging models in the long history of intercalation chemistry, namely the classical Rüdorff-Hofmann model proposed in 1938 and the pleated-layer domainmodified one in 1969, can explain the intercalation reaction phenomena and mechanism logically. Taking the landmark potassium-intercalation reaction of graphite as a model case and two advanced monolithic graphitic/graphenic carbon foams as model electrodes, here we have revealed that the electrochemical storage of potassium in graphitic/graphenic carbon (as that of lithium) obeys a simple interlayered centroid intercalation (ICIC) rule to achieve the staged potassium intercalation into each graphitic interlayer: $\mathrm{C} \rightarrow \mathrm{KC}_{72} \rightarrow \mathrm{KC}_{24} \rightarrow \mathrm{KC}_{8}$. Moreover, judging from the typical potassium-storage behaviors and crystal texture of graphitic electrodes, nitrogen doping and pre-embedded $\mathrm{K}$ atoms would enable incoming $\mathrm{K}^{+}$ions to perform fast pseudocapacitive diffusion in graphitic gallery. This study not only makes clear the basic K-storage mechanism and phenomena in graphitic carbon, but also establishes a more reasonable ICIC model for intercalation chemistry, and thus may help open a new research era for this field as well as graphitebased metal-ion batteries.
\end{abstract}




\section{Main Text}

Intercalation chemistry based on layered materials, including transition metal compounds and carbon especially graphite, is the operating basis of kinds of electrochemical energy-storage devices [1-3]. To explain related reaction mechanisms, phenomena, or composition and structure of the staged products, people have become accustomed to using the classical staging intercalation model proposed by Hofmann and his Ph.D. student Rüdorff in 1938 for $\mathrm{H}_{2} \mathrm{SO}_{4}$-graphite intercalation compounds (GICs) or the pleated-layer domain modified staging model updated by Daumas and Hérold in 1969 based on K-GICs [4-9]. However, as the theoretical basis of this field, such two models were built only according to the early X-ray diffraction (XRD) and highresolution (HR) cross-sectional transmission electron microscopy (TEM) characterizations about GICs of some molecules or atoms like $\mathrm{H}_{2} \mathrm{SO}_{4}, \mathrm{~K}, \mathrm{Br}_{2}$, and $\mathrm{FeCl}_{3}$ [4-7]. As a result, they have been considered to possess obvious logical contradiction in the model structure since the day of their birth $[5,6]$. For example, their most remarkable structural feature is that the adjacent intercalant layers for each Stage- $n$ GIC are separated periodically by $n$ layers of graphene sheets. It is consequently difficult to reasonably explain the evolution of the intercalants during stage change despite of the latter introduction of the pleated-layer structure [5, 6]. In fact, there are no reliable evidences for the existence of periodically distributed intercalant layers and widespread graphene stacking faults [10-12]. And neither of the two models has been verified by methodical crystallography and can endow its local structure with symmetrical surrounding (obligated to thermal stability and charge or electrostatic energy-distribution equilibrium) $[6,10]$. Researchers are always trying to solve this fundamental problem. For example, more and more studies have proposed that the intercalation/deintercalation of $\mathrm{H}_{2} \mathrm{SO}_{4}$ into graphite in fact mismatches the original theory $[11,12,18]$. Whereas, limited by material and technology, researchers just made some accidental modifications on the two problematic models according to their specific works, but could not give a reasonable explanation or even reach a scientific consensus on intercalation chemistry mechanism [6-18].

Recently, taking the virtue of a freestanding bilayer-graphene foam as model electrode, such as sufficient monolithic mass, absence of extrinsic interference from the as-applied substrates or other additives in lithium-ion batteries (LIBs), and against the ubiquitous aggregation and restacking of graphene sheets, we have established a new planar lithium-storage model to better describe and explain the staged Li-intercalation process into graphenic/graphitic interlayer[9, 19, 20]. Even so, due to the distinct physiochemical features of charge carriers (such as $\mathrm{Li}^{+}, \mathrm{Na}^{+}, \mathrm{K}^{+}$, $\mathrm{Mg}^{2+}, \mathrm{Zn}^{2+}, \mathrm{Al}^{3+}$, and anions) as well as the applied electrolyte (such as ether, alkylcarbonate, lactone, and ketal), different ions would exhibit different intercalation behaviors and processes into graphitic interlayer $[6,8,9]$. Hence, it is crucially necessary to make clear the intercalation chemistry of various ions from either a scientific or practical point of view. As mentioned above, the updated pleated-layer domain (D-H) model was built mainly on the study of K-GICs. Therefore, it is significantly important to revisit and clarify the K-storage mechanism of graphitic carbon. In fact, as a strong competitor to the commercial LIB techniques (in particular for stationary or grid applications requiring low cost $[21,22]$ ), K-ion batteries (KIBs) have inspired a new wave of research since 2015, when the state-of-the-art graphite anode in LIBs $\left(372 \mathrm{mAh} \mathrm{g}^{-1}\right.$ in theoretical capacity by forming the Stage-I Li-GIC $\mathrm{LiC}_{6}$ ) was proved to be also applicable in $\mathrm{KIBs}(279 \mathrm{mAh}$ $\mathrm{g}^{-1}$ in theoretical capacity by forming $\mathrm{KC}_{8}$ as the Stage-I K-GIC) [8, 9, 21-27]. However, despite of the employ of a variety of advanced ex-situ and in-operando characterization techniques (e.g., Raman spectroscopy and XRD) and the first principle density functional theory (DFT) calculations $[17,21,22,28-30]$, researchers even disagree on the staged compositions of the major K-GICs as a function of charge-discharge potential (let alone their crystalline structures under the primal D- 
$\mathrm{H}$ model), such as $\mathrm{KC}_{36} \leftrightarrow \mathrm{KC}_{24} \leftrightarrow \mathrm{KC}_{8}$ [21], $\mathrm{KC}_{24} \leftrightarrow \mathrm{KC}_{16} \leftrightarrow \mathrm{KC}_{8}$ [28], $\mathrm{KC}_{72} \leftrightarrow \mathrm{KC}_{24} \leftrightarrow \mathrm{KC}_{8}$ $[22,29], \mathrm{KC}_{84-72} \leftrightarrow \mathrm{KC}_{36} \leftrightarrow \mathrm{KC}_{24} \leftrightarrow \mathrm{KC}_{8}[30]$, and $\mathrm{KC}_{60} \leftrightarrow \mathrm{KC}_{48} \leftrightarrow \mathrm{KC}_{36} \leftrightarrow \mathrm{KC}_{24} / \mathrm{KC}_{16} \leftrightarrow \mathrm{KC}_{8}$ [17]. The unclear K-storage mechanism of graphite has become a macronosia for both intercalation chemistry and KIBs [7-9, 27].

Inspirited by the above, here we have comparatively studied the electrochemical K-storage behaviors of graphitic/graphenic carbon by employing two suitable freestanding graphene foams as model electrodes (refer to their advantages mentioned above) [19, 22, 29-34], including a trace nitrogen-doped multilayer-graphene foam ( 20-40 layers of graphene sheets, denoted as NGF) and a pristine fewlayer-graphene foam ( 2-4 layers of graphene sheets, denoted as GF) [19, 31]. Consistent well with previous reports, the $\mathrm{N}$ dopant can endow NGF with much better K-storage performance by enhancing its local K-adsorption capacity, electron conductivity, and ionic diffusion $[22,26,29,35]$. However, we find that the greatly lowered interface resistance and the $\mathrm{K}$ atoms pre-intercalated into the interlayer (priority at $\mathrm{N}$-doping sites $[22,36]$ ) should also be vital reasons for the superior rate and cycling performances of NGF. More important, it turns out that the two freestanding foam electrodes follow a similar interlayered centroid intercalation (ICIC) rule rather than the two historical staging theories to achieve the regular $\mathrm{K}$ storage, as follows: $\mathrm{C}$ (graphite) $\rightarrow \mathrm{KC}_{72} \rightarrow \mathrm{KC}_{24} \rightarrow \mathrm{KC}_{8}$. This study not only clarifies the electrochemical K-storage mechanism of graphitic carbon for KIBs [26, 27], but also confirms the versatility of the ICIC model in elucidating the graphite intercalation chemistry [7-9].

\section{Results}

\section{Materials characterization}

The monolithic NGF and GF electrodes, $\sim 30 \mu \mathrm{m}$ thick after removing the 3D nanoporous Ni template with ligament diameters at $300 \mathrm{~nm}-500 \mathrm{~nm}$ (Fig. 1a and Supplementary Figs 1 and 2), are both synthesized via chemical vapor deposition (CVD) processes using pyridine and benzene as the precursors $[19,31]$, respectively. In specialty, X-ray photoelectron spectroscopy (XPS) was applied to determine the amount and bonding configurations of the doped $\mathrm{N}$ elements in NGF. Based on the quantitative analysis results from the $\mathrm{C} 1 \mathrm{~s}$ (with the $\mathrm{C}-\mathrm{C}$ concentration at $\sim 81 \%$ ) and $\mathrm{N}$ 1s XPS spectra as well as the energy dispersive spectra (EDS), the total N/C ratio is estimated to be ca. 1/119 (Fig. 1b and Supplementary Fig. 2a-c). By deconvolution of the $\mathrm{N}$ 1s spectrum (Fig. 1b(ii)), the three peaks at binding energies of 399.4, 400.0, and $401.5 \mathrm{eV}$ can be indexed to pyridinic, graphitic/pyrrolic, and oxidic $\mathrm{N}$ species $[27,31,37,38]$, respectively. Thus, the small amount of graphic N (<30\% in considering of the co-presence of pyrrolic N; Fig. 1b(iii)) may imply that most of the $\mathrm{N}$ elements tend to be doped at edges or defects [22, 26, 27, 32-39]. This inference can be supported by the chemical mappings of electron energy-loss spectroscopy (EELS) reported previously [31], as well as the subsequent HR-TEM image of the basal plane of NGF showing only a few mono defects (Fig. 1f). By the way, many DFT simulations [24-26, 35, 36, $38,40-42]$ have demonstrated that, except that graphitic $\mathrm{N}$ sites would exhibit adverse impact for $\mathrm{K}$ adsorptions, other $\mathrm{N}$-doped sites (including N, O-dual doped sites [25]) can result in a local electron deficiency with a particularly high affinity for electron and thus significantly enhanced attraction to a nearby $\mathrm{K}$ atom $[22,35]$.

Despite of the N doping, the strong $G$ and $2 D$ Raman bands at 1685.4 and $2694.8 \mathrm{~cm}^{-1}$ (Fig. 1c), together with the following XRD (Fig. 1d) and TEM-related characterization results (Fig. 1eh), can confirm that NGF still possesses high-quality graphitic or graphenic crystal structure as that of GF. Differently, its much stronger $D$ band at $\sim 1354.4 \mathrm{~cm}^{-1}$ (with the $I_{D} / I_{G}$ ratios at 0.56 and 0.21 for NGF and GF, respectively) should imply that, the $\mathrm{N}$ doping process leads to more lattice defects. The defects of GF are considered to mainly come from the $\mathrm{sp}^{3} \mathrm{C}$ species locating at the 
structural edge planes, grain boundaries, or geometrical curvatures of the graphene sheets stripped from Ni substrate $[19,31,33]$. But for NGF, in view of the above XPS-based results, the significantly increased concentration of defective sites should be associated with both the similar structural defects enhanced by $\mathrm{N}$ doping and the homogenously dispersed and partially oxidized $\mathrm{N}$ atoms (refer to the inset of Supplementary Fig. 2a, c, Supplementary Fig. 3, and the EELS mapping images in refs. 31 and 34) [26]. This situation is also inflected in the mismatch on the measured interlayer spacing by the XRD and TEM-based techniques (with different test ranges to reflecting bulk or marginal-area structure information). The former indicates that the interlayer spacing (namely the $d_{002}$ value) of NGF is centered at $3.35 \AA$, as with the pristine AB-stacking GF sample possessing a more sharp (002) peak (Fig. 1d), while it is varied from 3.4 to $3.7 \AA$ by the latter (Fig. 1e-i and Supplementary Fig. 1f) [19,30]. Notwithstanding, the in-plane lattice spacings (e.g., $\sim 0.21$ and $0.12 \mathrm{~nm}$ for the $\{0110\}$ and $\{1210\}$ faces) are hardly changed and close to those of pristine graphene (Fig. 1d, e, g, h, i(iii) and Supplementary Fig. 3). That is, the trace N doping (< 0.3 at\% for graphitic $\mathrm{N}$ specie) doesn't change the basic honeycomb structure of pristine graphene foam, but it does modify its interlayer spacing in particular at edge plane [26, 33, 38]. By the way, the number of graphene layers at ca. 20-40 is sufficient to let NGF (Fig. 1f and Supplementary Fig. 1f and see its characteristic Raman spectrum in Fig. 1c) achieve a specific capacity close to that of graphite [19]. In addition, some pleated-layer defects can be clearly observed from the cross section namely (002) edge plane of a single multilayer-graphene sheet constructing NGF (Fig. 1f and i(iv) and Supplementary Fig. 3). Both of these stacking faults and here the $\mathrm{N}$-doping defects are believed to benefit $\mathrm{K}^{+}$ions to enter the graphitic interlayers but not affect the exploration of intrinsic K-storage mechanism of graphitic carbon.

\section{Electrochemical potassium-storage performances}

The K-storage performances of the freestanding NGF and GF electrodes were investigated using K-ion half-cells, where $\mathrm{K}$ metal foil was used as the counter electrode and $1 \mathrm{M}$ potassium bis(fluorosulfonyl)imide (KFSI) dissolved in ethylene carbonate/diethyl carbonate as the electrolyte. Galvanostatic charge-discharge (GCD) measurements (Fig. 2a-d and Supplementary Fig. 4) and cyclic voltammograms (CVs, Fig. 2e, f and Supplementary Figs 5-7) were firstly performed to determine their K-storage performances in terms of specific capacity and capacitance, respectively. In line with the previous report [22], the NGF material shows much higher K-storage capability to the fresh defective GF in various aspects, including the maximum reversible capacity/capacitance (ca. $326 \mathrm{mAh} \mathrm{g}^{-1} / 1284 \mathrm{~F} \mathrm{~g}^{-1}$ for NGF vs. $81 \mathrm{mAh} \mathrm{g}^{-1} / 241 \mathrm{~F} \mathrm{~g}^{-1}$ for GF, Fig. 2a, b, e), rate performances (Fig. 2e and Supplementary Figs 4c and 5i), and long-term cycling stability as well as Coulombic efficiency (Fig. 2c, d). Considering the edge locations of most of the extrinsic $\mathrm{N}$-doping defects and the intrinsic $\mathrm{sp}^{3} \mathrm{C}$ structural defects for here the specific graphene foam materials [24-26], as well as the entrance (namely the edge (002) planes) for $\mathrm{K}^{+}$ ions to diffuse into their galleries, the reason for such performance differences are considered as follows [22]: (i) trapping $\mathrm{K}$ atoms $\left(\mathrm{K}^{+}+\mathrm{e}^{-} \rightarrow \mathrm{K}^{0}\right)$ at the marginal $\mathrm{N}$-dopant sites of NGF [25, 36] would undoubtedly further enlarge the entrance to facilitate the $\mathrm{K}$ storage; (ii) the edged $\mathrm{C}$ defects with dangling bonds (refer to Fig. 1b(i) and Supplementary Fig. 2a), however, can only further shrink or reduce the relatively narrow entrances of GF to compromise the $\mathrm{K}$ storage. The much lowered interface resistance and improved electron transfer capability of NGF than that of GF makes this explanation more reasonable and comprehensive (Fig. 3a, b and refer to their Nyquist plots in Supplementary Fig. 8a, b). Although the two cells possessed similarly low ohmic resistances ( $<5 \Omega$ and originating from electrode, electrolyte, and separator) and high initial interface resistances ( $\sim 6280 \Omega \mathrm{cm}^{2}$ before working), the subsequently total interface resistances for NGF, dominated by the surface solid electrolyte interphase (SEI; generated during the initial 
discharge-charge process), became much smaller than that for GF (Fig. 3a). Their DC impedance $R$ values at each current density (Fig. 3b) further confirm this observation and the above deduction, which rose from ca. $0.20 \mathrm{k} \Omega \mathrm{cm}^{2}$ at $10 \mathrm{~A} \mathrm{~g}^{-1}$ to $1.26 \mathrm{k} \Omega \mathrm{cm}^{2}$ at $0.01 \mathrm{~A} \mathrm{~g}^{-1}$ for NGF and from 1.05 $\mathrm{k} \Omega \mathrm{cm}^{2}$ at $0.4 \mathrm{~A} \mathrm{~g}^{-1}$ to $3.07 \mathrm{k} \Omega \mathrm{cm}^{2}$ at $0.005 \mathrm{~A} \mathrm{~g}^{-1}$ for $\mathrm{GF}$, respectively. Therefore, it is quite necessary and crucial for an energy-storage electrode to possess simultaneously good electronic and ionic transfer capabilities [43]. The super steady K-storage capacities of another independent NGF electrode, which ran at varied current densities for hundreds and thousands of cycles (Fig. 2c), also illustrate this point. While, the GF electrode's capacity seemed to gradually increase until approaching to the theoretical value of trilayer graphene $\left(186 \mathrm{mAh} \mathrm{g}^{-1}\right)$ after cycling at $8.2 \mathrm{~mA} \mathrm{~g}^{-}$ ${ }^{1}$ for 100 cycles $\left(\sim 175 \mathrm{mAh} \mathrm{g}^{-1}\right.$, Fig. $2 \mathrm{~d}$ and Supplementary Fig. 4d). Judging from the Coulombic efficiencies always $<95 \%$ (Fig. 2d) and the formation reason of SEI, this phenomenon is associated with the ever-diminishing dangling organic groups as indicated above. As a result, more entrances will be generated to facilitate the diffusion of $\mathrm{K}^{+}$ions into graphitic galleries. By the way, to help make clear the K-storage mechanism of graphitic carbon, we stopped the above cycling test of 2\# NGF at discharged $0.20 \mathrm{~V}$ to obtain its Stage-II potassiated product (Supplementary Figs 9 and 10), which will be discussed in detail in Fig. 4.

\section{Electrochemical potassium-storage kinetics}

To fully understand the K-storage (vs. Li-storage) behavior of NGF and GF, the $b$ values, a common and important kinetic parameter, were obtained based on the $\mathrm{CV}$ curves at scan rates below $0.2 \mathrm{mV} \mathrm{s}^{-1}$ (Fig. 2f and Supplementary Figs 6 and 7) and the power-law relation $\left(i=a v^{b}\right.$ ) between $\mathrm{CV}$ peak current $(i)$ and scan rate $(v)[19,44]$. Generally, a $b$ value at 1.0 stands for a capacitive charge storage achieved by fast ion diffusion/adsorption on the surface, when the value at 0.5 refers to a typical battery reaction controlled by slow ion solid-state diffusion. Thus, judging from the obtained $b$ values for the distinguished redox peaks at $0.60-0.001 \mathrm{~V}$ (when the Stage-III $\mathrm{K}-\mathrm{GIC}$ has already formed), the kinetics diffusion behavior of $\mathrm{K}^{+}$ions in the potassiated GF are quite similar to that of $\mathrm{Li}^{+}$ions in lithiated bilayer graphene [19]. That is, the concentration of $\mathrm{K}$ atoms embedded in the graphitic interlayer intrinsically determines the intercalation kinetics of $\mathrm{K}^{+}$ ions, and the pseudocapacitive diffusivity of incoming $\mathrm{K}^{+}$ions will decrease as this concentration gradually increases. However, the cathodic and anodic $b$ values (i.e., 0.80 and 0.78 ) corresponding to the phase change between Stage-I and Stage-II K-GICs are both larger than the counterparts for lithiated bilayer graphene (i.e., 0.67 and 0.77) [19]. That is, the diffusion of $\mathrm{K}^{+}$ions in $\mathrm{KC}_{24}$ 's interlayer looks easier than that of $\mathrm{Li}^{+}$ions in $\mathrm{C}_{14} \mathrm{LiC}_{14}$ 's interlayer, as simultaneously reflected by their Bode diagrams (Supplementary Fig. 8c-f). Such an interesting phenomenon is possibly associated with the different atomic and ionic sizes of $\mathrm{K}$ and $\mathrm{Li}$ (with the diameters of $\mathrm{K}^{0} / \mathrm{K}^{+}$and $\mathrm{Li}^{0} / \mathrm{Li}^{+}$at 4.54/2.76 $\AA$ and 3.04/1.52 $\AA$, respectively [8]) and thus the remaining stereoscopic space in $\mathrm{K}$ or $\mathrm{Li}$ atoms-filled graphitic gallery. Given the approximate in-plane compositions of the potassiated NGF and GF at Stages I and II, the general variation trend for each $b$ value, increasing with the decrease of scan rate (Supplementary Figs 6c, d and 7c, d), further supports the above inference and in particular the significant role of interlayer spacing.

To further verify the significance of interlayer spacing (proportional to the amount of $\mathrm{K}$ atoms embedded in graphene interlayer $[21,27]$ ), the well-known Galvanostatic intermittent titration (GITT) technique $[17,24,25]$ was applied to determine the chemical diffusion coefficients of $\mathrm{K}^{+}$ ions $\left(D_{\mathrm{K}}\right)$ in the NGF electrode at GCD current densities of 0.1 and $0.2 \mathrm{~A} \mathrm{~g}^{-1}$ (Fig. $3 \mathrm{c}, \mathrm{d}$ and Supplementary Fig. 11), respectively. As is expected, as a function of the state of charge (SOC), the $D_{\mathrm{K}}$ values evaluated at $0.1 \mathrm{~A} \mathrm{~g}^{-1}$ are always larger than the counterparts at $0.2 \mathrm{~A} \mathrm{~g}^{-1}$ (ranged between $10^{-9}-10^{-7} \mathrm{~cm}^{2} \mathrm{~s}^{-1}$ at SOC $<60 \%$ ), well demonstrating the above kinetics analysis. In addition, the two groups of $D_{\mathrm{K}}$ values basically share the same evolution trends to those of the $b$ 
values, in particular noticing how they change during the depotassiation or charging processes (e.g., with $b$ values for the ever-present anodic peak continually decreasing from 0.96 below 0.2 $\mathrm{mV} \mathrm{s}^{-1}$ to 0.48 or even 0.25 between $0.2-50 \mathrm{mV} \mathrm{s}^{-1}$ [27]; Supplementary Fig. 6c). Meanwhile, the slight change in the $D_{\mathrm{K}}$ values at SOC $<60 \%$ (between $1.0 \times 10^{-9}$ and $2.0 \times 10^{-9} \mathrm{~cm}^{2} \mathrm{~s}^{-1}$ ) should imply that the $\mathrm{K}^{+}$ion-intercalation kinetics has a close correlation with the distribution density of $\mathrm{K}$ atoms. Obviously, it is difficult to use the two classical staging models to explain such a kinetics behavior.

\section{Electrochemical potassium-storage mechanism}

Judging from the GCD curves of NGF and GF (Fig. 2a, b), there are mainly three distinguished slope regions accounting for three-stage K-intercalation reactions [26, 27]. Specifically, according to the discharge curve of NGF at the smallest current density of $0.01 \mathrm{~A} \mathrm{~g}^{-}$ 1 (Supplementary Fig. 6c), the capacities at the end of each potential slope (i.e., 0.40, 0.20, and $0.001 \mathrm{~V}$ ) are ca. 48, 144, and $327 \mathrm{mAh} \mathrm{g}^{-1}$, respectively. Considering the deviation of $\sim 48 \mathrm{mAh}$ $\mathrm{g}^{-1}$ between the maximum capacity and the theoretical $279 \mathrm{mAh} \mathrm{g}^{-1}$ for $\mathrm{KC}_{8}$ (associated with the $\mathrm{N}$-doping and $\mathrm{C}$ defective sites [19, 22]), the actual capacity contributed by $\mathrm{K}$ intercalation at 0.20 $\mathrm{V}\left(\sim 96 \mathrm{mAh} \mathrm{g}^{-1}\right)$ should imply that the Stage-II K-GIC phase is close to the widely reported $\mathrm{KC}_{24}$ of $93 \mathrm{mAh} \mathrm{g}^{-1}$ (Fig. 4a). To further confirm the staged K-intercalation process, analysis was carried out in terms of the NGF and GF's CV curves between 0.60 and $0.001 \mathrm{~V}$ at low enough scan rates (Fig. 2f, g and Supplementary Fig. 6a, b). As the scan rate decreases from 20 to $2 \mu \mathrm{V} \mathrm{s}^{-1}$, the initial two obvious pairs of CV redox peaks gradually split into five pairs for both NGF and GF, similar to the lithiated-bilayer graphene case and characteristic of the staged quasi-equilibrium phase transformations of $\mathrm{KC}_{x}$ [19]. Despite of this fact, here these split peaks are still treated as one in view of their high-degree overlap (standing for rapid phase transformation [17]). Accordingly, the Stage-II phase is estimated to approach $\mathrm{KC}_{24}$ as well according to the average area ratios of the two integrated peaks at various scan rates (Fig. $2 \mathrm{~g}$; refer to the $\mathrm{KPF}_{6}$ electrolyte-based CV curves in Supplementary Fig. 13). Theoretically, such a composition also corresponds to a very stable structure judging from the geometric locations of its $\mathrm{K}$ atoms on the graphenic carbon matrix (Supplementary Fig. 12a). Furthermore, the $\mathrm{K}$ atoms of $\mathrm{KC}_{8}$ are found to locate right in the centroid of three adjacent $\mathrm{K}$ atoms of $\mathrm{KC}_{24}$, highly consistent with the $\mathrm{Li}$-storage mode in bilayer graphene [19]. Thus, we predict that the Stage-III K-GIC is $\mathrm{KC}_{72}$ of $31 \mathrm{mAh} \mathrm{g}^{-1}$ by such an ICIC rule (Figs $2 \mathrm{~g}$ and $4 \mathrm{a}$ ), agreeing well with the previous reports and the GCD result mentioned above. Consequently, all the analyses indicate that the elementary $\mathrm{K}$-intercalation process in graphitic interlayer should be $\mathrm{C}$ (graphite) $\rightarrow \mathrm{KC}_{72} \rightarrow \mathrm{KC}_{24} \rightarrow \mathrm{KC}_{8}$ in theory (Fig. 4a).

Notwithstanding, it is still uncertain whether the classical pleated-layer staging model proposed 50 years ago is suitable to describe the $\mathrm{K}$-intercalation scene in the $c$ direction [4-6, 10, 12, 19]. To well address this historic problem, here we have carefully studied the crystal structure of the representative Stage-II K-NGF phase (close to $\mathrm{KC}_{24}$ in composition; Supplementary Figs 9 , 10 , and 13) by multiple TEM-based advanced characterization techniques (Fig. 4b-e and Supplementary Figs 14-20 and Video 1). Similar to what happened in the lithiated-bilayer graphene foam [19], high-energy electron beam irradiation (EBI) also seriously changed the initial distribution of $\mathrm{K}$ atoms embedded in the graphitic interlayers (Fig. 4b), as reflected by those everchanging SAED patterns (Fig. 4c, d and Supplementary Fig.14) and HR-TEM images (Fig. 4e and Supplementary Figs 15-20) [26, 27]. Differently, due to the much larger diameter of K atom (4.54 $\AA$ ) than that of $\mathrm{Li}$ atom $\left(3.04 \AA\right.$ ) versus that of $\mathrm{C}_{6}$-ring unit $(2.84 \AA)$, a greatly enhanced expansion in graphene honeycomb lattice was observed in this case (up to $\sim 29 \%$ vs. $\sim 8 \%$ for Stage-II $\mathrm{C}_{14} \mathrm{LiC}_{14}$ [19]; Fig. 4c, d and Supplementary Figs 12 and 16-20). Meanwhile, most of the K atoms would be squeezed away from the region under electron beam, rather than being forced into 
graphenic matrix like Li atoms [19]. Relative evidences include the lattice ripples (ca. 1-4 nm apart from each other) caused by regular movement of the interbedded $\mathrm{K}$ atoms (Fig. 4e and Supplementary Fig. 15a, h), some individual $\mathrm{K}$ atoms residual in the irradiation region (Supplementary Fig. 15h, i), the regular $d_{002}$ variations in different regions (Fig. 4c-e and Supplementary Figs 15d-f, 16, and 17) [27], the gradual emergence of the coexistence scene of the intrinsic and expanded graphenic carbon lattices (Fig. 4c, d and Supplementary Figs 16-20), and the generation of crystal lattices of the K-atom intercalation layers [19] which should come from the derived $\mathrm{KC}_{8}$ or $\mathrm{KC}_{16}$ structure under $\mathrm{EBI}$ (Fig. 4b-d and Supplementary Figs 12 and 1620). Thus, based on these EBI-driven structure changes, the $\mathrm{KC}_{24}$ 's intrinsic configuration can be ascertained reasonably as shown in Fig. $4 \mathrm{a}$, where the adopted $d_{002}$ values have been widely confirmed by XRD technology $[17,21]$. By the way, the sharp $d_{002}$ increase from Stage II to Stage I (ca. $4.39 \AA$ for $\mathrm{KC}_{24}$ and $5.40 \AA$ for $\mathrm{KC}_{8}$ ) can well illustrate the stacking configuration change of $\mathrm{K}-\mathrm{GICs}$ from $\mathrm{A} R \mathrm{~B}$ to $\mathrm{A} R \mathrm{~A}$. In particular, it can be seen clearly from the quickly captured HRTEM images of random locations that all the Stage-II K-NGF's interlayers are roughly uniform and equal in spacing (Fig. 4e). And as confirmed simultaneously by the XRD pattern with a single (002) peak $\left(d_{002}=4.87 \AA\right.$; Fig. 4f and Supplementary Fig. 9d), there are no empty graphitic galleries to periodically separate two adjacent $\mathrm{K}$ intercalant layers, completely different from what the two classical staging models presented (see the inset of Fig. 4f). In fact, it is just because of the undifferentiated intercalation behaviors of $\mathrm{K}^{+}$ions in each graphitic/graphenic interlayer that the NGF and GF samples possessing quite different K-storage capacities can exhibit analogous CV features (Fig. 2 and Supplementary Figs 5-7). Thus, here the ICIC model (Fig. 4a) can not only logically explain the above kinetics phenomena, but also can reasonably describe and even predict the staged structure and composition of K-GICs.

\section{Discussion}

Neither of the two classical staging models in the history of intercalation chemistry (as well as their derivatives) can explain the potassium-storage process of graphite without logical defects. With enlarged marginal interlayer spacing caused by trace nitrogen doping but still high crystalline quality, the freestanding multilayer NGF electrode (Fig. 1) is expected to help solve this problem. Using a defective fewlayer graphene foam GF as counterpart (Fig. 1c, d) and according to the statistical electrochemical data recorded at enough small current density or slow CV scan rate (Fig. 2), we find that the two monolithic electrodes in fact demonstrate similar K-intercalation processes by following a regular ICIC rule: $\mathrm{C} \rightarrow \mathrm{KC}_{72} \rightarrow \mathrm{KC}_{24} \rightarrow \mathrm{KC}_{8}$ (Fig. 4). And, against the theoretical basis of the two historical staging models [6-9], the TEM characterizations imply that there are no empty graphene layers observed in the typical Stage-II phase (Fig. 4e, f) despite of the mere existence of pleated-layer stacking faults in the initial sample (Fig. 1f, i). The kinetics analyses (Fig. 3c, d) further indicate that, the diffusion of $\mathrm{K}^{+}$ions in graphitic interlayer are closely related to the distribution concentration of the embedded $\mathrm{K}$ atoms and tend to be capacitive due to the larger interlayer spacing, consistent well with the ICIC theory. On the other hand, the comparative analyses about the K-storage performances (Fig. 2a-e) and impedances (Fig. 3a, b) of the two model electrodes also illustrate that, unimpeded ion diffusion at the edge plane and good electronic transmission at the SEI-dominated interface are both necessary for a high-performance graphitic anode in KIBs. Thus, in view of the well-documented N-doping effects, these results in turn confirm that N-doped graphitic carbon foam can make an ideal anode material for KIBs [26].

In summary, taking the advantages of monolithic graphene foam electrodes, here we have

revealed the fundamentally staged $\mathrm{K}$-intercalation mechanism for graphitic carbon as follows: $\mathrm{C}$ $\rightarrow \mathrm{KC}_{72} \rightarrow \mathrm{KC}_{24} \rightarrow \mathrm{KC}_{8}$. It is found that the electrochemical storage of potassium in graphitic/graphenic carbon obeys a similar ICIC rule to that of lithium, rather than the two historic 
staging models widely used in graphite intercalation chemistry. Meanwhile, it is clarified that the superior K-storage performances and capacitive kinetics of nitrogen-doped multilayer graphene foam can be attributed to its enlarged interlayer spacing and lowered interface resistance, which can make it a qualified mixed conductor for both ions and electrons [43] when working in KIBs. This study not only confirms the versatility of the ICIC model in elucidating the staging Kintercalation phenomena in graphitic/graphenic interlayer, which may help open a new era of research in intercalation chemistry $[6-9,12,17]$, but also makes clear the design approach to promote graphitic carbon for better KIBs.

\section{Methods}

Materials Preparation. Both the nitrogen-doped multilayer graphene foam (NGF) and fewlayer graphene foam (GF) were synthesized via a CVD process employing nanoporous $\mathrm{Ni}(n p-\mathrm{Ni})$ as template and liquid organic compound with small molecule as graphene precursor. Thereinto, the $n p$-Ni template was prepared by dealloying $50 \mu \mathrm{m}$-thick $\mathrm{Ni}_{30} \mathrm{Mn}_{70}$ foil in $2.0 \mathrm{M}\left(\mathrm{NH}_{4}\right)_{2} \mathrm{SO}_{4}$ solution for $10 \mathrm{~h}$, which during the preparation was placed at the center of a quartz tube $(\varphi 30 \times \varphi 27 \times 1000$ $\mathrm{mm}$ ) of the CVD furnace.

The $n p$-Ni@NGF material was prepared at $800{ }^{\circ} \mathrm{C}$ by injecting gasified pyridine $\left(\mathrm{C}_{5} \mathrm{H}_{5} \mathrm{~N}\right)$ in the gas flow of $\mathrm{Ar}(2500 \mathrm{sccm})$ and $\mathrm{H}_{2}(100 \mathrm{sccm})$ for 3 min using the method shown in ref. 31 . The np-Ni@GF material was prepared from gasified benzene $\left(\mathrm{C}_{6} \mathrm{H}_{6}, 30 \mathrm{~s}\right.$ for gas entry) by following the high-temperature-switched CVD procedures described in ref. 19, where the high and low temperatures were set at 950 and $850{ }^{\circ} \mathrm{C}$, respectively. And the target freestanding NGF and GF materials were obtained through dissolving $\mathrm{Ni}$ substrate in $2.0 \mathrm{M} \mathrm{HCl}$, repeatedly waterisopropanol washing, and the supercritical $\mathrm{CO}_{2}$ drying.

For the potassiated NGF sample, it was carefully taken down from its coin cell disassembled in an argon-filled glove box and then fully rinsed by pure ethylene carbonate (EC)/diethyl carbonate (DEC) electrolyte.

Structure and Composition Characterization. Microstructural and chemical characterizations were performed using a field-emission transmission electron microscope (JEOL JEM-2100F and F200, $200 \mathrm{kV}$ ) and scanning electron microscope (FEI Apreo S LoVac, 2 kV; JEOL JIB-4600F, $15 \mathrm{kV}$ ) equipped with X-ray energy-dispersive spectrometer. The Raman spectra measurements were performed on a micro-Raman spectrometer (Renishaw InVia RM 1000) with excitation laser wavelength of $514.5 \mathrm{~nm}$. The XRD patterns were collected on an X-ray diffractometer (SmartLab) using $\mathrm{Cu} K \alpha$ radiation and nickel filter $(\lambda=0.15406 \mathrm{~nm})$, and the operating voltage and current were $40 \mathrm{kV}$ and $30 \mathrm{~mA}$. The XPS spectra were measured by using an X-ray Photoelectron Spectroscopy (XPS, AXIS ultra DLD, Shimazu) with an Al K $\alpha$ (mono) anode at $150 \mathrm{~W}$ in a vacuum of $10^{-7} \mathrm{~Pa}$.

Electrochemical Measurements. Coin 2032-type test cells were assembled in a high-purity argon-filled glove box $\left(\mathrm{H}_{2} \mathrm{O}<0.5 \mathrm{ppm}, \mathrm{O}_{2}<0.5 \mathrm{ppm}\right.$, SegoStation) with $1 \mathrm{M}$ potassium bis(fluorosulfonyl)imide (KFSI) dissolved in EC and DEC (EC:DEC $=1: 1 \mathrm{v} / \mathrm{v}$ ) as the electrolyte, the freestanding graphene foams directly as the working electrodes, fresh potassium foil as the counter electrode and Whatman glass fiber as the separator. Galvanostatic charge/discharge (GCD), cyclic voltammetry (CV), and galvanostatic intermittent titration (GITT) measurements were carried out at room temperature on LAND CT2001A and Neware battery testing systems and Ivium electrochemical workstation. The electrochemical impedance spectrum and Bode phase diagram were measured on Ivium electrochemical workstation simultaneously at varied open 
circuit voltages with a sinusoidal signal in a frequency range from $100 \mathrm{kHz}$ to $0.1 \mathrm{~Hz}$ at an amplitude of $5 \mathrm{mV}$.

\section{Data availability}

The authors declare that the major data supporting the findings of this study are available within the paper and its Supplementary Information. Extra data are available from the authors upon reasonable request.

\section{References}

1. Freunberger, S. A. True performance metrics in beyond-intercalation batteries. Nature Energy 2, 17091 (2017).

2. Yang, C. Y., et al. Aqueous Li-ion battery enabled by halogen conversion-intercalation chemistry in graphite. Nature 569, 245-250 (2019).

3. Lin, M.-C., et al. An ultrafast rechargeable aluminium-ion battery. Nature 520, 324-328 (2015).

4. Hoffmann, U. \& Rüdorff, W. The formation of salts from graphite by strong acids. Trans. Faraday Soc. 34, 1017-1021 (1938).

5. Daumas, N. \& Hérold, A. Relations between the elementary stage and the reaction mechanisms in graphite insertion compounds. Compt. Rend. Acad. Sci. Ser. C Sci. Chim. 268, 373-375 (1969).

6. Hérold, A. Crystallo-chemistry of carbon intercalation compounds. Phys. Chem. Mater. Layered Struct. 6, 323-421 (1979).

7. Lerf, A. Storylines in intercalation chemistry. Dalton Trans. 43, 10276-10291 (2014).

8. Li, Y. Q., Lu, Y. X., Adelhelm, P., Titirici, M. \& Hu. Y.-S. Intercalation chemistry of graphite: alkali metal ions and beyond. Chem. Soc. Rev. 48, 4655-4687 (2019).

9. Wang, G.; Yu, M. H \& Feng X. L. Carbon materials for ion-intercalation involved rechargeable battery technologies. Chem. Soc. Rev. 50, 2388-2443 (2021).

10. Timp, G. \& Dresselhaus, M. S. The ultramicrostructure of commensurate graphite intercalation compounds. J. Phys. C Solid State Phys. 17, 2641-2651 (1984).

11. Dimiev, A. M., et al. Direct real-time monitoring of stage transitions in graphite intercalation compounds. ACS Nano 7, 2773-2780 (2013).

12. White, E. R., Lodico, J. J. \& Regan, B. C. Intercalation events visualized in single microcrystals of graphite. Nat. Commun. 8, 1969 (2017).

13. Yazami, R. \& Reynier, Y. Thermodynamics and crystal structure anomalies in lithiumintercalated graphite. J. Power Sources 153, 312-318 (2006).

14. Sethuraman, V. A., Hardwick, L. J., Srinivasan, V. \& Kostecki, R. Surface structural disordering in graphite upon lithium intercalation/deintercalation. J. Power Sources 195, 3655-3660 (2010).

15. He, H., Huang, C., Luo, C. -W., Liu, J. -J. \& Chao, Z. -S. Dynamic study of Li intercalation into graphite by in situ high energy synchrotron XRD. Electrochim. Acta 92, 148-152 (2013).

16. Allart, D., Montaru, M. \& Gualous, H. Model of lithium intercalation into graphite by potentiometric analysis with equilibrium and entropy change curves of graphite electrode. $J$. Electrochem. Soc. 165, A380-A387 (2018). 
17. Liu, J. L. et al. Unraveling the potassium storage mechanism in graphite foam. Adv. Energy Mater. 9, 1900579 (2019).

18. Besenhard, J. O. et al. Anodic oxidation of graphite in $\mathrm{H}_{2} \mathrm{SO}_{4}$ dilatometry - in situ X-ray diffraction - Impedance spectroscopy. Synth. Met. 7, 185-192 (1983).

19. Ji, K. M. et al. Lithium intercalation into bilayer graphene. Nat. Commun. 10, 275 (2019).

20. Zhong, K. H. et al. Adsorption and ultrafast diffusion of lithium in bilayer graphene: $\mathrm{Ab}$ initio and kinetic Monte Carlo simulation study. Phys. Rev. B 99, 155403 (2019).

21. Jian, Z. L., Luo, W. \& Ji, X. L. Carbon electrodes for K-ion batteries. J. Am. Chem. Soc. 137, 11566-11569 (2015).

22. Share, K. et al. Role of nitrogen-doped graphene for improved high-capacity potassium ion battery anodes. ACS Nano 10, 9738-9744 (2016).

23. Fredenhagen, K. \& Cadenbach, G. Die bindung von Kalium durch kohlenstoff. Z. Anorg. Allg. Chem. 158, 249-263 (1926).

24. $\mathrm{Xu}, \mathrm{Y}$. et al. Highly nitrogen doped carbon nanofibers with superior rate capability and cyclability for potassium ion batteries. Nat. Commun. 9, 1720 (2018).

25. Zeng, S. F. et al. Boosting the potassium storage performance of carbon anode via integration of adsorption-intercalation hybrid mechanisms. Nano Energy 73, 104807 (2020).

26. Wu, X. et al. Advanced carbon-based anodes for potassium-ion batteries. Adv. Energy Mater. 9, 1900343 (2019).

27. Zhang, W. L. et al. Graphitic nanocarbon with engineered defects for high-performance potassium-ion battery anodes. Adv. Funct. Mater. 29, 1903641 (2019).

28. Luo, W. et al. Potassium ion batteries with graphitic materials. Nano Lett. 15, 7671-7677 (2015).

29. Share, K., Cohn, A. P., Carter, R. E. \& Pint, C. L. Mechanism of potassium ion intercalation staging in few layered graphene from in situ Raman spectroscopy. Nanoscale 8, 16435-16439 (2016).

30. Liang, H.-J. et al. Staging Na/K-ion de-/intercalation of graphite retrieved from spent Li-ion batteries: in operando X-ray diffraction studies and an advanced anode material for $\mathrm{Na} / \mathrm{K}$-ion batteries. Energy Environ. Sci. 12, 3575-3584 (2019).

31. Ito, $\mathrm{Y}$. et al. Bicontinuous nanoporous $\mathrm{N}$-doped graphene for the oxygen reduction reaction. Adv. Mater. 26, 4145-4150 (2014).

32. Bernardo, I. D. et al. Two-dimensional hallmark of highly interconnected three-dimensional nanoporous graphene. ACS Omega 2, 3691-3697 (2017).

33. Bernardo, I. D. et al. Topology and doping effects in three-dimensional nanoporous graphene. Carbon 131, 258-265 (2018).

34. Kumatani, A. et al. Chemical dopants on edge of holey graphene accelerate electrochemical hydrogen evolution reaction. Adv. Sci. 6, 1900119 (2019).

35. Wang, J. et al. Fabrication of porous carbon with controllable nitrogen doping as anode for high-performance potassium-ion batteries. ChemElectroChem 6, 3699-3707 (2019).

36. Zhou, X. F. et al. Three-dimensional ordered macroporous metal-organic framework single crystal-derived nitrogen-doped hierarchical porous carbon for high-performance potassiumion batteries. Nano Lett. 19, 4965-4973 (2019).

37. Figueras, $M$. et al. Correcting flaws in the assignment of nitrogen chemical environments in N-doped graphene. J. Phys. Chem. C 123, 11319-11327 (2019).

38. Wang, H. H. et al. Nitrogen configuration dependent holey active sites toward enhanced $\mathrm{K}^{+}$ storage in graphite foam. J. Power Sources 419, 82-90 (2019).

39. Wang, X. R. et al. N-doping of graphene through electrothermal reactions with ammonia. Science 324, 768-771 (2009). 
40. Li, D. P. et al. Facile fabrication of nitrogen-doped porous carbon as superior anode material for potassium-ion batteries. Adv. Energy Mater. 8, 1802386-1802395 (2018).

41. Chang, X. Q. et al. Ultrahigh nitrogen doping of carbon nanosheets for high capacity and long cycling potassium ion storage, Adv. Energy Mater. 9, 1902672 (2019).

42. Ruan, J. F. et al. Rational construction of nitrogen-doped hierarchical dual-carbon for advanced potassium-ion hybrid capacitors. Adv. Energy Mater. 10, 1904045 (2020).

43. Chen, C.-C. \& Maier, J. Decoupling electron and ion storage and the path from interfacial storage to artificial electrodes. Nat. Energy 3, 102-108 (2018).

44. Simon, P., Gogotsi, Y. \& Dunn, B. Where do batteries end and supercapacitors begin? Science 343, 1210-1211 (2014).

\section{Acknowledgments}

This work was sponsored by the National Natural Science Foundation of Tianjin (20JCQNJC01280), the Peiyang Scholars programs of Tianjin University (2020XRG-0013 and 2019XRY-0048), the National Natural Science Foundation of China (21905201), and JSPS Grantin-Aid for Scientific Research on Innovative Areas "Discrete Geometric Analysis for Materials Design" (Grant Numbers JP20H04628). We thank the Instrument analysis and testing center at School of Chemical Engineering in Tianjin University, University of Tsukuba, and Advanced Institute for Materials Research (AIMR) in Tohoku University for experiment assistance. Author contributions

K.M.J. conceived the idea and wrote the manuscript. K.M.J., K.L.H., and Y.I. prepared and characterized the graphene foam materials. K.M.J. carried out the electrochemical measurements and analyzed all the experimental data. K.M.J. and Y.H.S. constructed the structure model. C.L. and Y.Z. contributed to the battery assembly and disassembly. Z.Z.L., J.W., M.Q.W., and X.Y.L. helped carry out parts of the material characterization and data analysis. Y.I., J.S., M.M.C., J.F., and C.Y.W. helped carry out the experiments. Y.I. and S.Z. commented on the manuscript.

\section{Additional information}

Supplementary information is available in the online version of the paper. Reprints and permissions information is available online. Correspondence and requests for materials should be addressed to K.M.J.. The authors declare no competing financial interests. The corresponding author would like to dedicate this paper to his newborn daughter. 

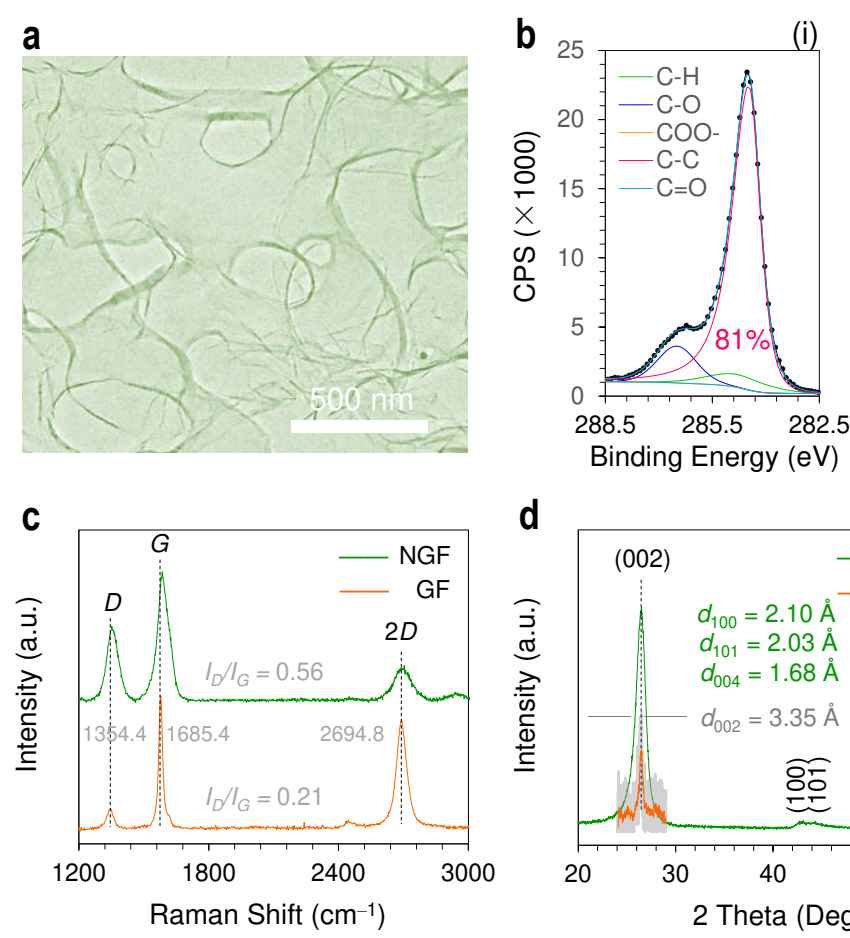

f

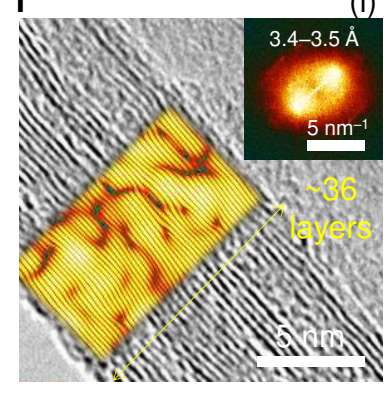

g

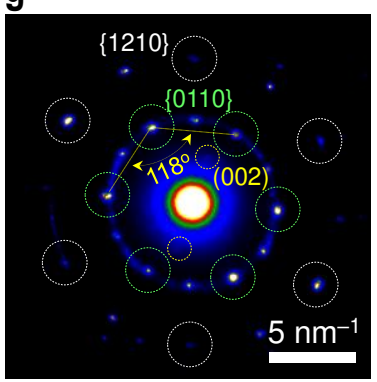

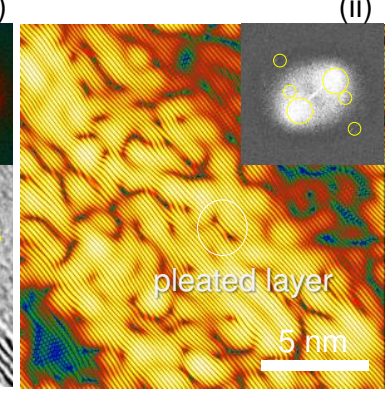

h

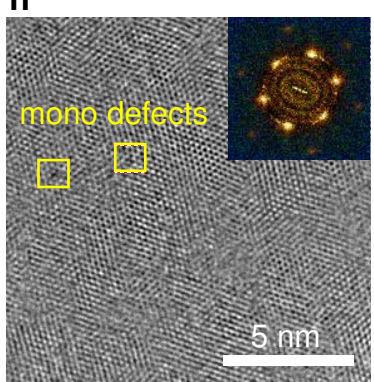

d

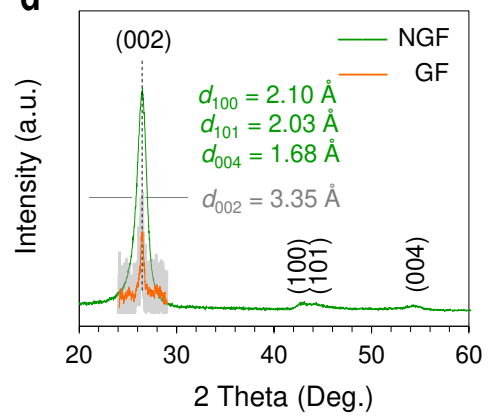

(ii) $\mathbf{i}$
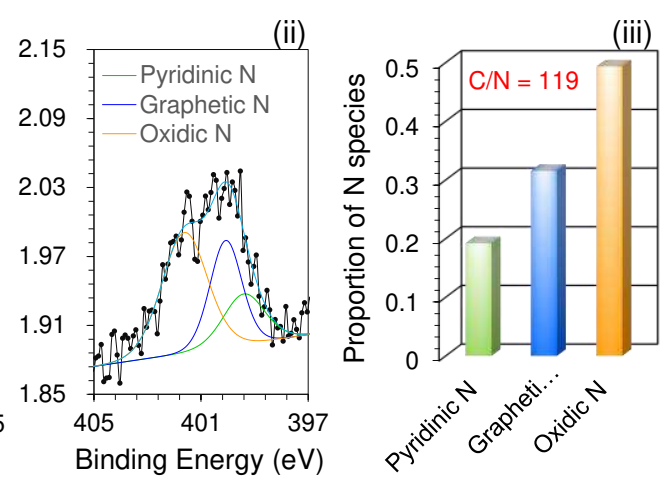

e

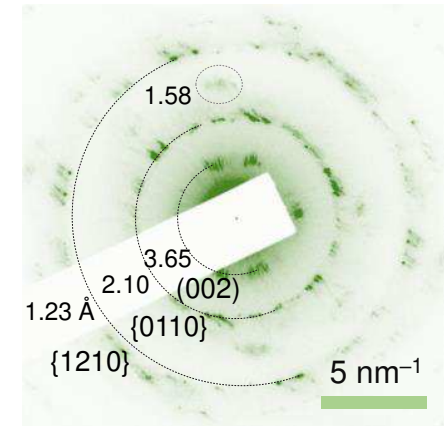

(i)

(ii)
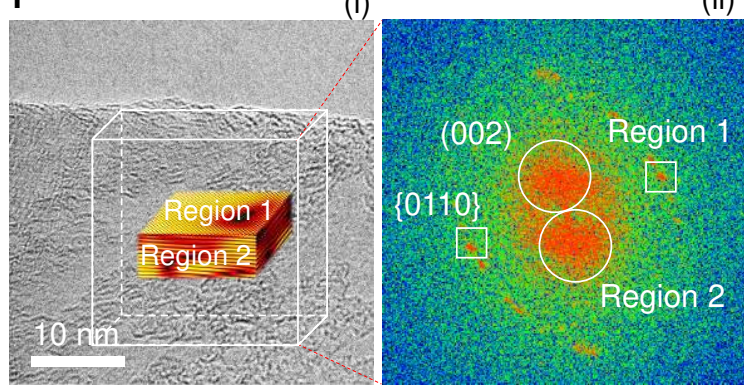

(iii)

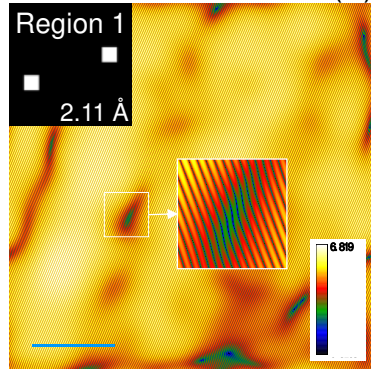

(iv)

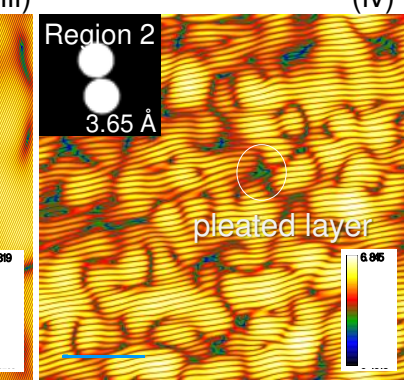

Fig. 1 | Microstructure and composition of the 3D NGF material. a, TEM image of NGF. b, C 1s and N1s XPS spectra and the contents of N species. c, d, Comparative Raman spectra (c) and XRD patterns (d) of NGF and GF. e, g, SAED patterns from marginal multilayer region (e) and flat fewlayer region (g) of NGF. f, h, i, HR-TEM images from cross section (f), flat region (removing amorphous carbon through the average background subtraction filter) (h), and marginal area (i) of some single graphene sheets constructing the NGF foam, together with their corresponding FFT patterns (see the insets in $\mathbf{f}(\mathrm{i}, \mathrm{ii}), \mathbf{h}$, and $\mathbf{i}(\mathrm{ii})$ ) and some specific inverse FFT images (f(ii) and i(iii, iv)). Pleated-layer defects can be observed clearly from the cross section (f and $\mathbf{i}(\mathrm{iv}))$. The SAED and FFT pattens of non-regular hexagonal shape shown in $(\mathbf{g}-\mathbf{i})$ imply that their selected regions were not completely flat or the electron beam was not normal-incidence ${ }^{[19]}$. 

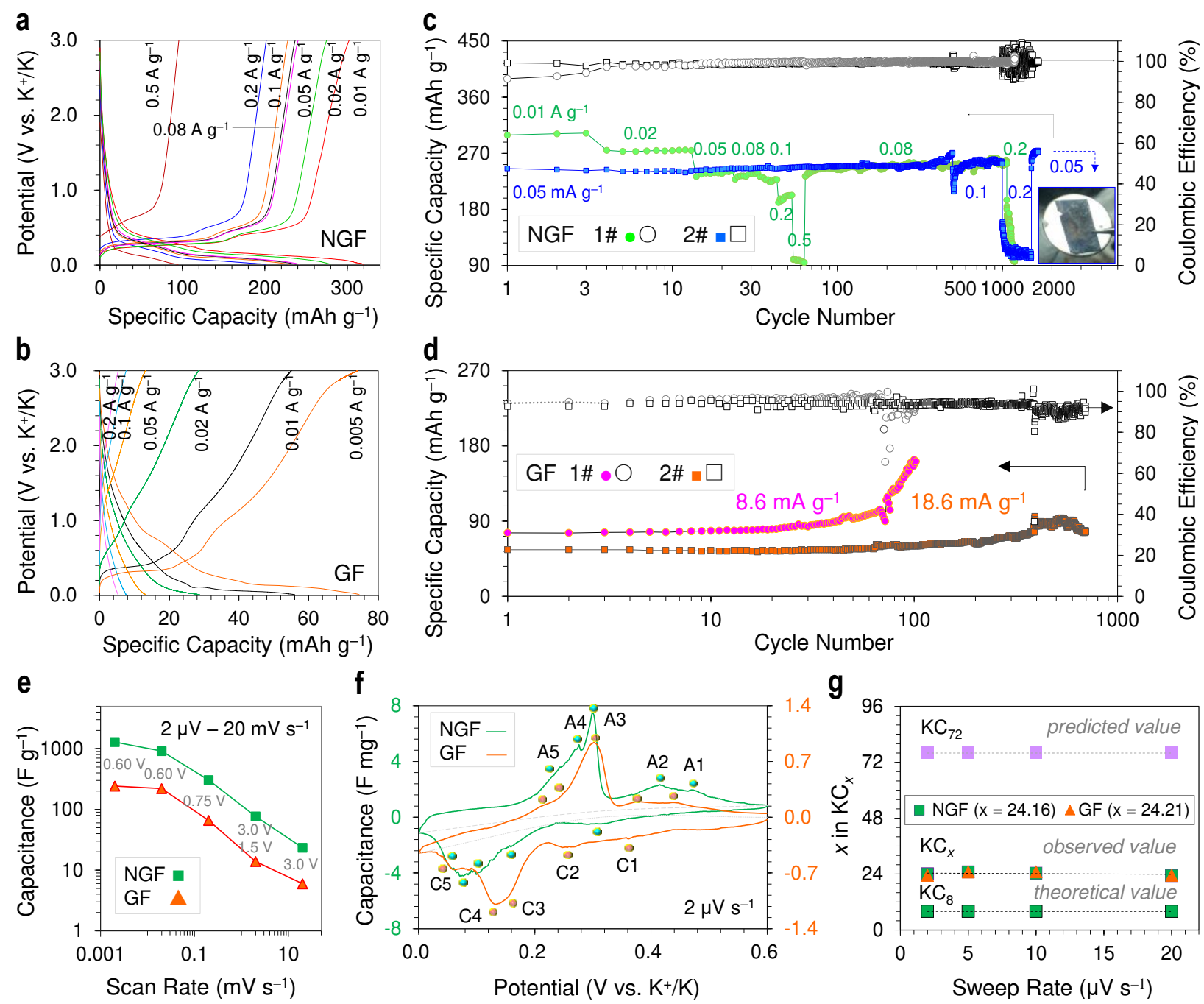

Fig. 2 Comparative electrochemical performances of the freestanding NGF and GF electrodes. a, b, GCD curves of NGF (a) and GF (b) at varied current densities. c, d, Cycling and rate performances of NGF (c) and GF (d). Two cells were assembled for either sample (1\# and 2\#) for the tests. The 2\# cell of NGF was disassembled to check the microstructure of its potassiated phase at Stage II. e, Specific capacitances of NGF and GF at CV scan rates varied from $20 \mathrm{mV} \mathrm{s}^{-}$ ${ }^{1}$ to $2 \mu \mathrm{V} \mathrm{s}^{-1}$. The values marked next the curves refer to the high-end cut-off potentials in calculating the two samples' specific capacitances (Supplementary Fig. 7a, b). f, CV curves at 2 $\mu \mathrm{V} \mathrm{s}^{-1}$ for NGF and GF, where C1-5 and A1-5 refer to the visible cathodic and anodic peaks on behalf of the K-intercalation/deintercalation reactions of $\mathrm{K}^{+}+\mathrm{e}^{-}+\mathrm{KC}_{x} \leftrightarrow \mathrm{KC}_{y}$, respectively. C1 and $\mathrm{C} 2$ are assigned to the formation of Stage-II $\mathrm{KC}_{24}$, and C3, C4, and $\mathrm{C} 5$ are corresponding to the formation of Stage-I $\mathrm{KC}_{8}$ together with the $\mathrm{K}$ storage at some defects. $\mathrm{g}$, Determination of the Stage-II $\mathrm{KC}_{x}$ products for NGF and GF by calculating CV peak areas at low CV scan rates (Supplementary Fig. 6a, b). The contributions of defective sites, ca. 15\% according to the specific capacity of $327 \mathrm{mAh} \mathrm{g}^{-1}$ at $0.01 \mathrm{~A} \mathrm{~g}^{-1}$ (Fig. 2a and Supplementary Fig. 4a-c) and the theoretical $279 \mathrm{mAh} \mathrm{g}^{-1}$, were excluded in the calculations. 
a

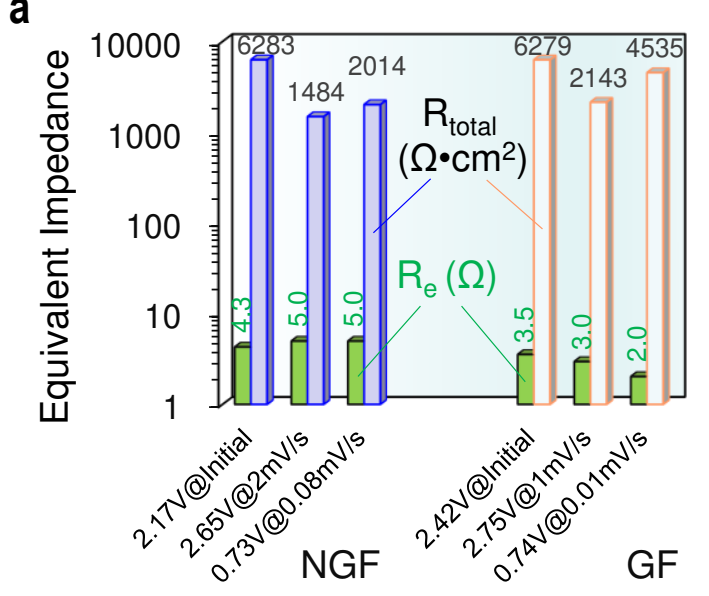

C

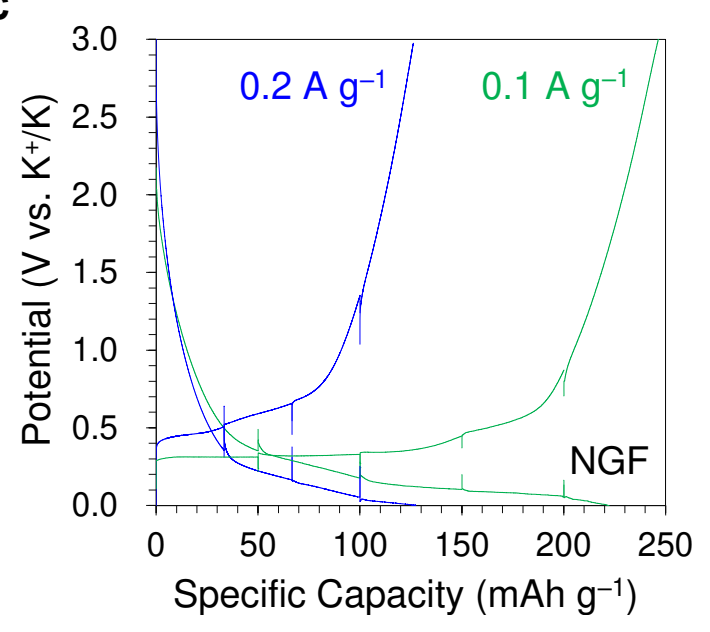

b

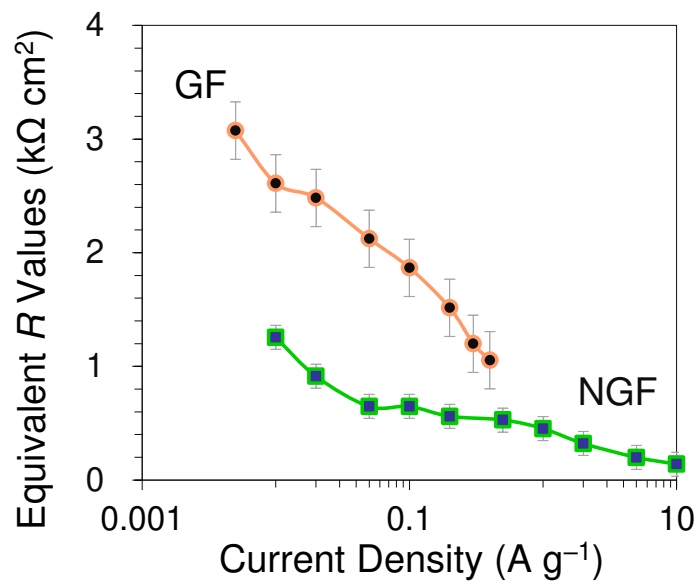

d

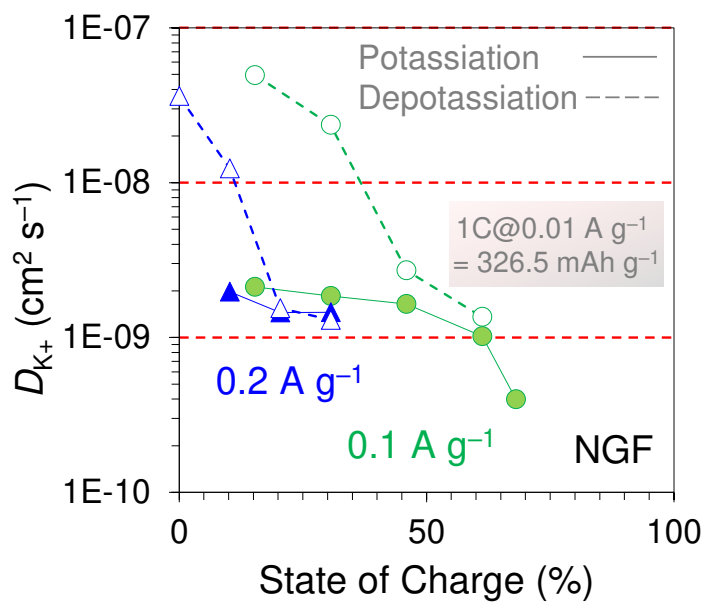

Fig. 3 | Electrochemical resistance of the NGF electrode and kinetics analysis about its Kstorage capability. a, Comparative AC equivalent resistances of NGF and GF based on the simulation of their Nyquist plots measured at different OCPs as a function of CV scan rates (Supplementary Fig. 5). $\mathrm{R}_{\mathrm{e}}$ (unit: $\Omega$ ) refers to the total Ohmic resistance from the electrode, electrolyte, and separator of the test cell, while $\mathrm{R}_{\text {total }}$ (unit: $\Omega \mathrm{cm}^{2}$ ) refers to the SEI-dominant interface resistances (originating from the SEI itself, the electrode-SEI interface, and the SEIsolution interface) and it is associated with the exposed surface of the specific electrode sample in the electrolyte ${ }^{[19]} \cdot \mathbf{b}$, Comparative DC equivalent resistances of NGF and GF based on the voltage drops at varied GCD current densities (refer to Fig. 2a, b). c, GITT profiles at 0.1 and $0.2 \mathrm{~A} \mathrm{~g}^{-1}$ for the NGF sample after a long-term GCD cycling test (refer to Fig. 2c and Supplementary Figs 9-11). d, Apparent chemical diffusion coefficients of $\mathrm{K}^{+}$ions in the NGF electrode as a function of SOC. The SOC values were calculated using the two sets of specific capacities obtained from (c) by assuming that the specific capacity of $326.5 \mathrm{mAh} \mathrm{g}^{-1}$ measured at $0.01 \mathrm{~A} \mathrm{~g}^{-1}$ (Fig. 2a and Supplementary Fig. $4 \mathrm{a}-\mathrm{c}$ ) was the maximum capacity of NGF. 

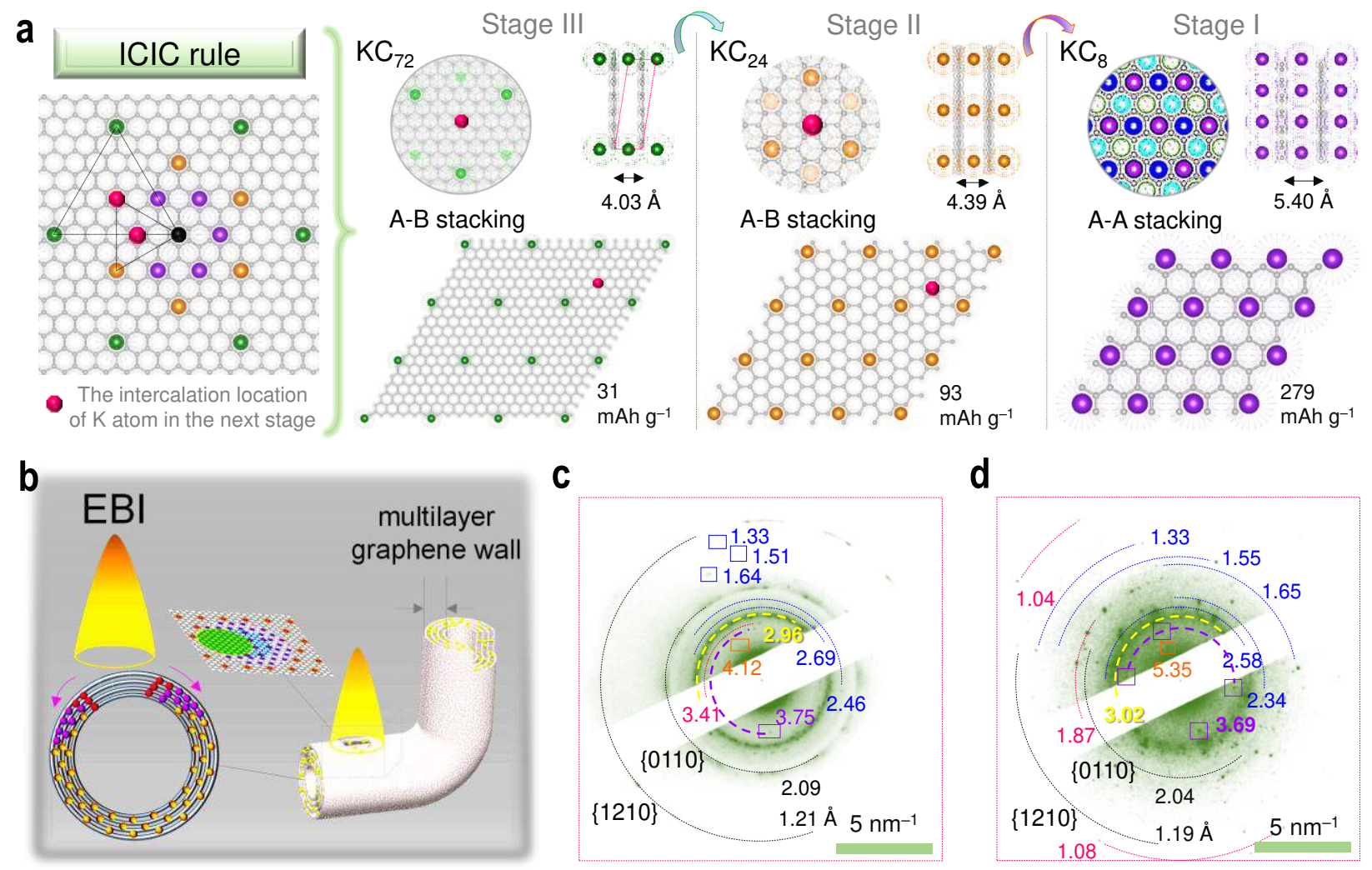

C

d
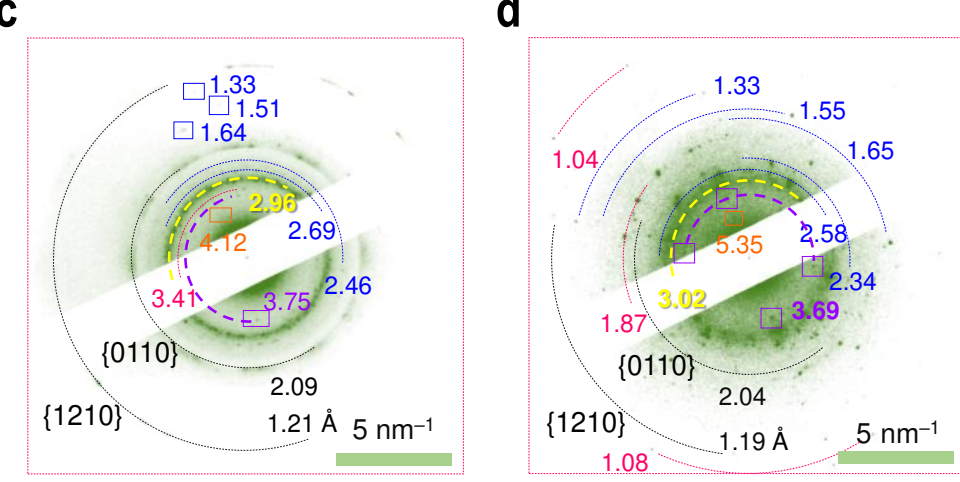

e
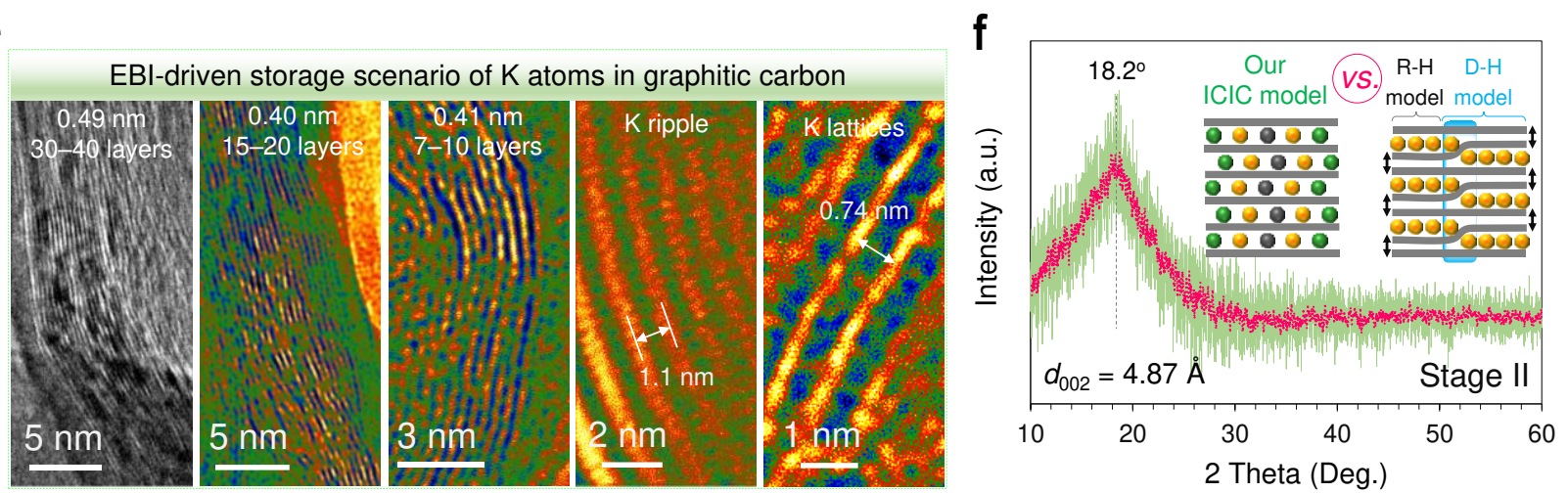

Fig. 4 | Determination of the electrochemical K-storage mechanism for graphitic/graphenic carbon based on the Stage-II K-NGF material potassiated at $0.2 \mathrm{~V}$. a, Schematic of the ICIC rule for the staged K-storage process into graphitic/graphenic carbon and the configuration diagrams for the three main K-GICs. b, Schematic of the EBI influence on the $c$-direction and planar distribution of $\mathrm{K}$ atoms storing at marginal area of curved multilayer graphene sheet (refer to Supplementary Figs 12 and 14-20). c, d, SAED patterns of the Stage-II K-NGF captured immediately (c) and after a long period of radiation (d; refer to Supplementary Fig. 14). e, HRTEM images captured from cross section or marginal area of the Stage-II K-NGF under EBI (refer to (b) and Supplementary Figs 15 and 20). f, XRD pattern of the Stage-II K-NGF (refer to Supplementary Fig. 9). The insets display the structure of the Stage-II $\mathrm{KC}_{24}$ by the ICIC rule and by the classical R-H and D-H models, respectively. 


\section{Supplementary Files}

This is a list of supplementary files associated with this preprint. Click to download.

- SAEDStagellKGICs.mp4

- SupplementaryinformationKMJI20210610.pdf 